\title{
Revisiting the PeVatron candidate MGRO J1908+06 with an updated H.E.S.S. analysis
}

\section{Kostunin, ${ }^{a, *}$ L. Mohrmann, ${ }^{b}$ E. de Ona Wilhelmi, ${ }^{a}$ V. Joshi, ${ }^{b}$ A. Mitchell, ${ }^{c}$ S. Ohm, ${ }^{a}$ B. Khélifi, ${ }^{d}$ L. Giunti ${ }^{d, e}$ and A. Sinha ${ }^{f}$ on behalf of the H.E.S.S. Collaboration} (a complete list of authors can be found at the end of the proceedings)

${ }^{a}$ DESY, D-15738 Zeuthen, Germany

${ }^{b}$ Friedrich-Alexander-Universität Erlangen-Nürnberg, Erlangen Centre for Astroparticle Physics, Erwin-Rommel-Str. 1, D 91058 Erlangen, Germany

${ }^{c}$ Max-Planck-Institut für Kernphysik, P.O. Box 103980, D-69029 Heidelberg, Germany

${ }^{d}$ Université de Paris, CNRS, Astroparticule et Cosmologie, F-75013 Paris, France

${ }^{e}$ Institute for Research on the Fundamental Laws of the Universe (IRFU), Commissariat à l'énergie atomique (CEA), Université Paris-Saclay, F-91191 Gif-sur-Yvette, France

${ }^{f}$ Laboratoire Univers et Particules de Montpellier, CNRS

Université de Montpellier, F-34090 Montpellier, France

E-mail: contact.hess@hess-experiment.eu

Detecting and studying galactic gamma-ray sources emitting very-high energy photons sheds light on the acceleration and propagation of cosmic rays presumably created in these sources. Currently, there are few sources emitting photons with energies exceeding $100 \mathrm{TeV}$. In this work we revisit the unidentified source MGRO J1908+06, initially detected by Milagro, using an updated H.E.S.S. dataset and analysis pipeline. The vicinity of the source contains a supernova remnant and pulsars as well as molecular clouds. This makes the identification of the primary source(s) of galactic cosmic rays as well as the nature of the gamma-ray emission challenging, especially in light of the recent HAWC and LHAASO detection of the high energy tail of its spectrum. Exploiting the better angular resolution as compared to particle detectors, we investigate the morphology of the source as well as its spectral properties.

$37^{\text {th }}$ International Cosmic Ray Conference (ICRC 2021)

July 12 th - 23rd, 2021

Online - Berlin, Germany

\footnotetext{
${ }^{*}$ Presenter
} 


\section{Introduction}

Mapping the sky in very-high-energy gamma-rays sheds light on the acceleration and propagation of Galactic cosmic rays, i.e. probing acceleration mechanisms in the vicinity of stellar objects, particle escape, etc. One of the most interesting questions is to search for PeVatrons - accelerators of cosmic-ray nuclei up to $1 \mathrm{PeV}$ (the cosmic-ray knee), studying their population and acceleration cutoff. The Galactic Center was the first detected PeVatron [1], recently H.E.S.S. detected another candidate HESS J1702-420 [2].

Modern high-altitude particle arrays focused on gamma-ray detection brought intriguing results recently: HAWC has published a list of galactic sources with emission above $56 \mathrm{TeV}$, where a number of them feature emission above $100 \mathrm{TeV}$ [3], LHAASO has published the first dozen of its ultrahigh energy gamma-ray sources [4]. Some of them have been previously detected by H.E.S.S., e.g. eHWC J1825-134 (LHAASO J1825-1326), which HESS resolved into two sources; HESS J1825-137 and HESS J1826-130, demonstrating its superior angular resolution [5, 6].

This works describes a new analysis of HESS J1908+063 (hereafter J1908+063), which was initially discovered by Milagro [7] and later confirmed by H.E.S.S. [8], HAWC (eHWC J1907+063) and LHAASO (LHAASO J1908+0621). Although the source was first detected more than a decade ago, its nature is still unidentified. However, recent investigations of this region with different instruments have brought new insights as to its nature. Besides recent results from ground gammaray detectors mentioned above, several multi-wavelength studies (including with Fermi-LAT and radio observations) have been conducted [9-11] since J1908+063 has last been studied with H.E.S.S. in context of its Galactic Plane Survey [12]. This progress motivates us to revisit this source using additional H.E.S.S. data not analysed previously with an updated reconstruction pipeline.

J1908+063 is spatially coincident with a number of stellar objects, which can potentially produce very-high-energy gamma rays. Two main candidates have been suggested as the sources of emission: PSR J1907+0602 [13] and SNR G40.5-0.5 [14] assuming pulsar wind nebula and molecular clouds interaction scenarios, respectively. Recently discovered PSR J1907+0631 [15], possibly associated with SNR G40.5-0.5, is also energetically viable for gamma-ray production. There are two more objects located at the northern edge of the source, which can also produce gamma rays and contribute to the J1908+063 emission: PSR J1906+0722 [16] and SNR 3C397 [17]. The summary of their properties is given in Tab. 1.

\begin{tabular}{lllll} 
Object & $d(\mathrm{kpc})$ & $t_{\text {age }}(\mathrm{kyr})$ & pulsar $\dot{E}(\mathrm{erg} / \mathrm{s})$ & SNR radio size \\
\hline PSR J1907+0602 [13] & $3.2 \pm 0.6$ & 19.5 & $2.8 \times 10^{36}$ & - \\
PSR J1906+0722 [16] & 1.91 & 49.2 & $1.02 \times 10^{36}$ & - \\
PSR J1907+0631 [15] & 7.9 & 11.2 & $5 \times 10^{35}$ & - \\
SNR G40.5-0.5 [14] & $5.5-8.5$ & $20-40$ & - & $22^{\prime}$ \\
SNR 3C397 [17] & $8-9$ & $1.35-5.3$ & - & $4.5^{\prime} \times 2.5^{\prime}$
\end{tabular}

Table 1: Objects in the vicinity of J1908+063, which can potentially produce very-high-energy gamma rays. The methods of age estimation are dependent on the object class. 


\section{Observation and analysis}

The High Energy Stereoscopic System (H.E.S.S.) is an array of four 12-m and one 28-m Imaging Atmospheric Cherenkov Telescopes (IACTs) located in the Khomas Highland in Namibia at an altitude of $1835 \mathrm{~m}$. It is capable of detecting VHE gamma-rays from energies of a few tens of $\mathrm{GeV}$ to $100 \mathrm{TeV}$. The data used in this work were acquired prior to the installation of the $28-\mathrm{m}$ telescope.

Compared to the latest H.E.S.S. publication including J1908+063 [12], the present work uses additional data acquired since the last publication, which adds about $50 \%$ more exposure (mostly in the northern part of the source). The resulting livetime for the central part of the source is almost 80 hours after applying quality cuts. For the low-level reconstruction we use events detected by four telescopes with maximum offset of $2^{\circ}$ from the center of the camera. Direction and energy reconstruction and gamma/hadron separation is performed with methods described in Refs. [18, 19].

The high-energy analysis is performed with gammapy v0.17 [20,21] with an energy threshold of $\sim 0.365 \mathrm{TeV}$ and three-dimensional model of residual hadronic background. For the crosscheck with previous H.E.S.S. results and newest ones obtained by Fermi-LAT [11], HAWC [3] and LHAASO [4], we fitted J1908+063 assuming single Gaussian component with spectrum described by power law. The preliminary fitted values are (only statistical errors are included): R.A. $=286.975^{\circ} \pm 0.024^{\circ}$, dec. $=6.432^{\circ} \pm 0.024^{\circ}$, sigma of Gaussian $\sigma=0.524^{\circ} \pm 0.018^{\circ}$. The spectrum described by the parametrization $\phi(E)=\phi_{0}(E / \mathrm{TeV})^{-\Gamma}$ has the following values (only statistical errors are included): $\phi_{0}=(1.02 \pm 0.05) \cdot 10^{-11} \mathrm{TeV}^{-1} \mathrm{~cm}^{-2} \mathrm{~s}^{-1}, \Gamma=2.294 \pm 0.027$. The comparison of morphological and spectral fits between different instruments is given in Fig. 1.
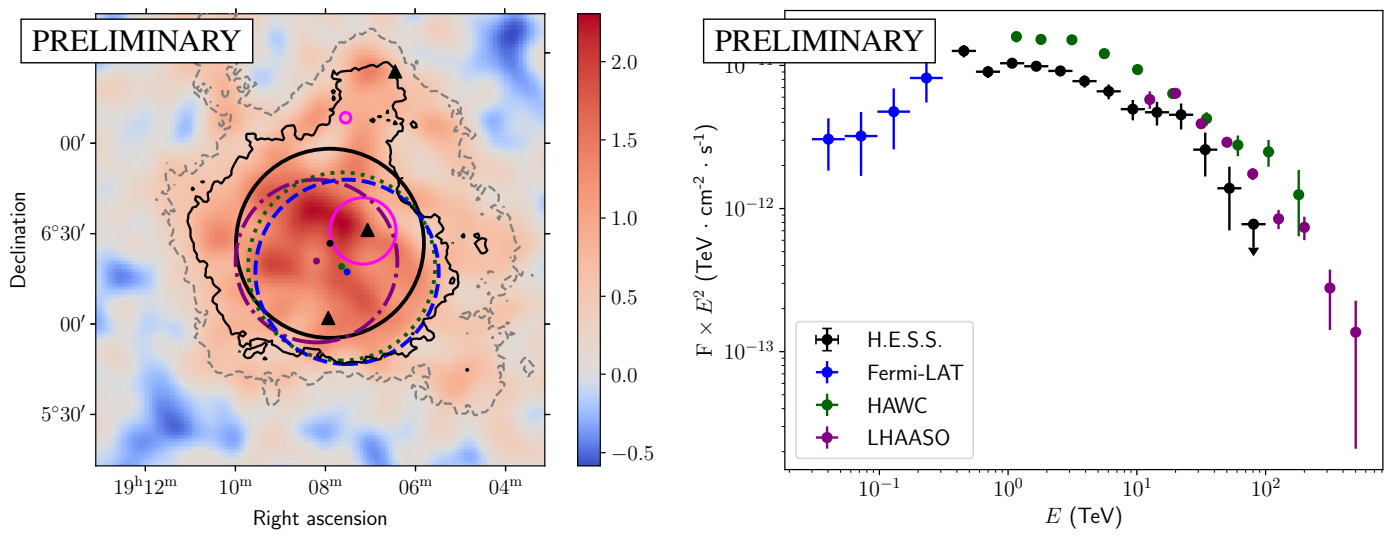

Figure 1: Comparison between single-component morphology and spectra reconstructed by different instruments with H.E.S.S. reconstruction obtained in this work. Left: H.E.S.S. excess map smoothed with radius of $0.07^{\circ}$. Gray dashed and black solid contours correspond to $3 \sigma$ and $5 \sigma$ Li-Ma significance, respectively. Circles correspond to morphology fits by H.E.S.S. (black solid with $r=\sigma_{\text {source}}$, 3D analysis with gammapy using Gaussian model), Fermi-LAT (blue dashed with $r=r_{\text {disk}}$, using disk model) [11], HAWC (green dotted with $r=\sigma_{\text {source }}$, using Gaussian model) [3] and LHAASO (purple dash-dotted radius corresponding to containment $68 \%$ of source) [4]. Triangles indicate pulsars, magenta circles indicate supernova remnants (see Tab. 1). Right: comparison of the spectra reconstructed by different instruments using spacial models described above. 
The next step in our analysis is to probe for energy-dependent features of the source. We have chosen six energy bins, four of which $-(0.365,0.649),(0.649,1.0),(1.0,1.78),(1.78,4.87) \mathrm{TeV}$ - contain roughly the same number of excess events (about 1100), and two high-energy regions: $E>4.87 \mathrm{TeV}$ (420 excess events) and $E>10 \mathrm{TeV}$ (190 excess events) still featuring significant detection of the source. The significance maps are given in Fig. 2. We have searched for dependence of single-component fit parameters on energy in these bins, however they stay constant within uncertainties. Taking into account the complex surroundings of J1908+063 we plan to perform morphology fits using different models and number of components.

In the present analysis we pre-defined three regions based on possible origins of the gamma-ray emission with astrophysical associations. Fig. 3 shows the geometry configuration of the regions and their spectra, while Tab. 2 shows the fitted parameters of power-law spectral model. There is indication of spectral difference across the region, but this remains to be confirmed with further tests.

As an intermediate result we produce the integral flux maps assuming a power law with a spectral index of 2.3 and show them in combination with radio measurements from Ref. [9], see Fig. 4. We see no striking spatial correlation between very-high-energy gamma-ray emission detected by H.E.S.S. and radio emission as detected in Ref. [9].

\section{Conclusion and discussion}

The vicinity of J1908+063 contains plenty of stellar objects as well as dense molecular clouds, which can generate very-high-energy gamma emission. In this work we have revisited this source with an extended H.E.S.S. dataset and new analysis pipeline using gammapy for high-level analysis, which has given a significant detection in six energy bins and allowed for the testing of energydependent morphology.

The single-component fit obtained using 3D analysis in gammapy is in very good agreement with the previously published H.E.S.S. results. It is worth noting that the spectral reconstruction by this new analysis technique does not require containment correction (as described in Ref. [12]), since it takes into account the entire source and assumes pre-defined morphology (Gaussian shape in our case), which is important for studying of very extended sources.

This is an another cross-check and validation of gammapy, a toolset for the future gamma-ray observatories, which has started to be used in H.E.S.S. recently [2, 23, 24].

\begin{tabular}{lllllll} 
Region & R.A. & Dec. & Radius & Excess & $\phi_{0}\left(10^{-12} \mathrm{TeV}^{-1} \mathrm{~cm}^{-2} \mathrm{~s}^{-1}\right)$ & $\Gamma$ \\
\hline A & $286.87^{\circ}$ & $6.12^{\circ}$ & $0.4^{\circ}-0.8^{\circ}$ & 1413.9 & $1.89 \pm 0.12$ & $2.43 \pm 0.06$ \\
B & $286.92^{\circ}$ & $6.17^{\circ}$ & $0.2^{\circ}$ & 498.3 & $0.637 \pm 0.071$ & $2.12 \pm 0.07$ \\
C & $286.65^{\circ}$ & $7.20^{\circ}$ & $0.2^{\circ}$ & 197.6 & $0.265 \pm 0.061$ & $2.12 \pm 0.13$
\end{tabular}

Table 2: Preliminary geometrical and spectral properties for the regions A, B, C reconstructed for the power-law model. Confidence regions include statistical uncertainties only. 

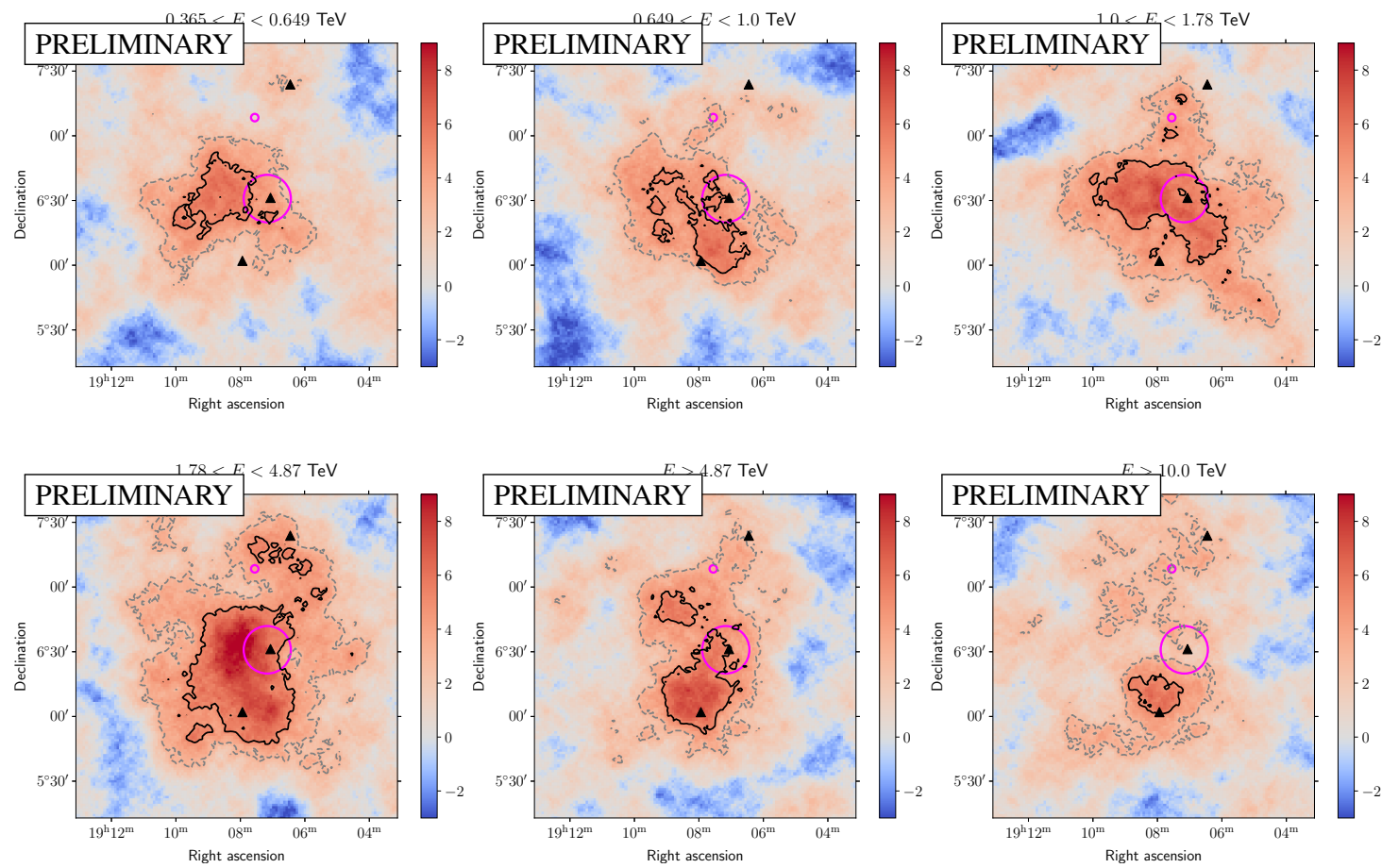

Figure 2: Significance maps reconstructed for 6 energy bands using Li-Ma definition [22]. Gray dashed and black solid contours correspond to 3 and 5 sigma significance, respectively. All color scales are defined with the bounds $(-3 ; 8)$ sigma in order to simplify comparison. See Fig. 1 for further details on the legend.
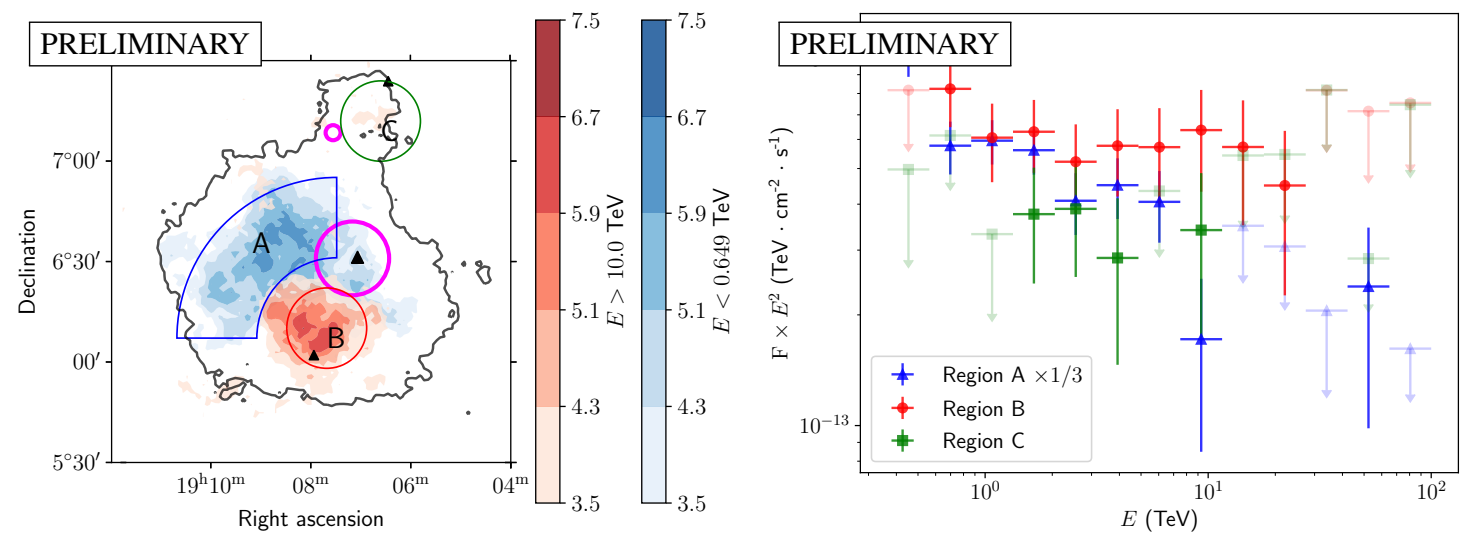

Figure 3: Comparison of spectra reconstructed from the different parts of the source. Region A (blue segment) in the North-West part of the source, roughly equally distanced from pulsars and supernova remnant, Region B (red circle) in the southern part of the source nearby PSR J1907+0602 and Region C (green circle) in the northern part of the source nearby PSR J1906+0722. Left: configuration of the regions, black contour represents $5 \sigma$ Li-Ma significance contour for all-energy emission, blue and line contours are Li-Ma significance contours for low-energy $(E<0.649 \mathrm{TeV})$ and high-energy $(E>10 \mathrm{TeV})$ emission. Right: Spectra extracted from three regions normalized by solid angle. Spectrum for Region A is normalized for the solid angle to ease the comparison. See Fig. 1 for further details on the legend. 


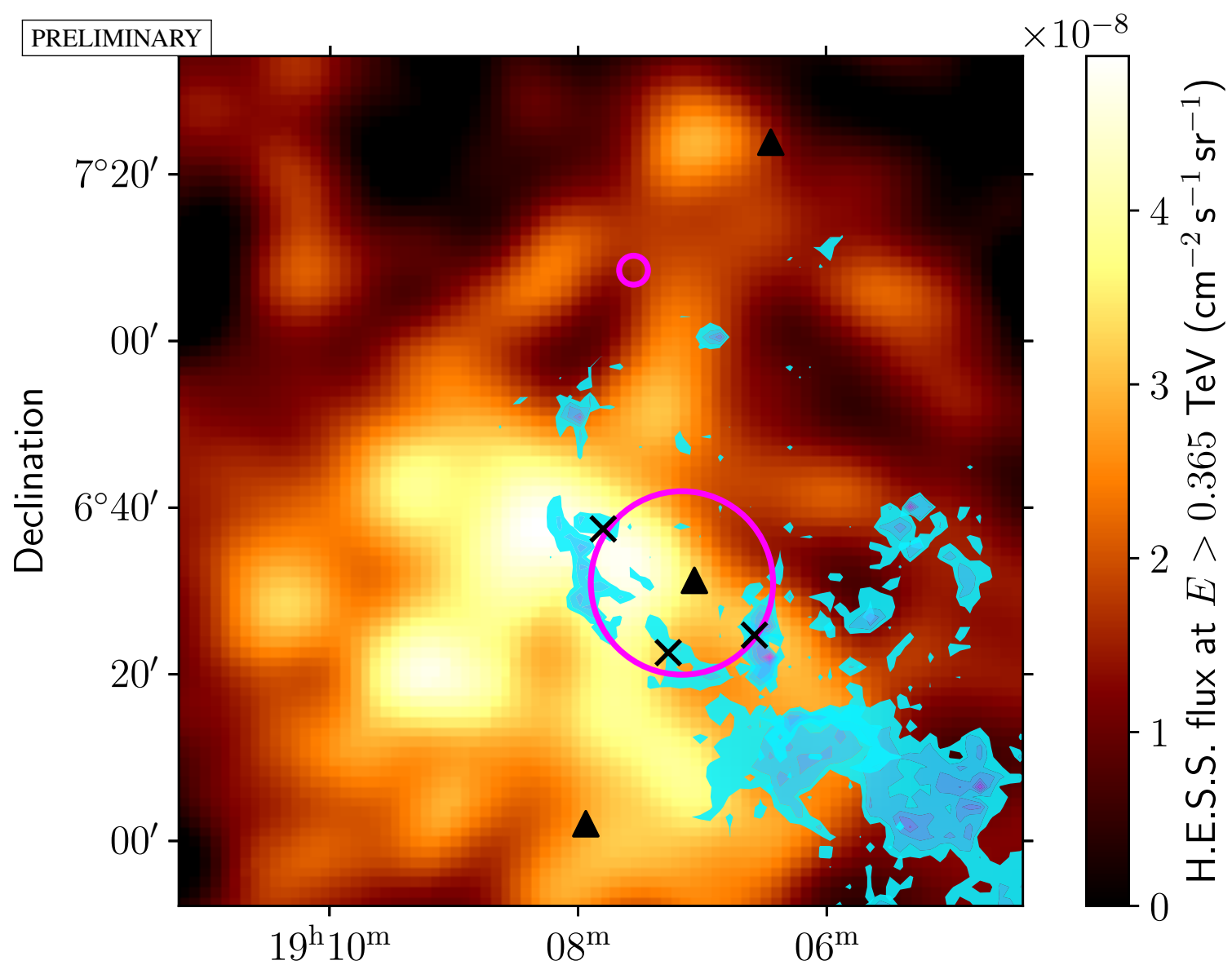

Right ascension

Figure 4: H.E.S.S. flux maps combined with ${ }^{12} \mathrm{CO}$ measurements and possible interaction with SNR G40.5-0.5 as suggested in Ref. [9]. Filled contours (cyan) show molecular clouds at different distances, crosses indicate possible hadronic interactions (see Ref. [9]).

The extended exposure for J1908+063 and surroundings sheds more light on the composition of this densely populated region. We see hints on emission in the Northern part of the source. This emission can be potentially associated with PSR J1906+0722 and SNR 3C397. To resolve possible contributions from these sources we will perform additional morphology fits, since all sources are in close proximity and featuring contamination from each other. On the other hand these data are not sufficient to claim the origin of emission in the central part of the source, resolve more than one component or see energy-dependent morphology with high statistical significance. The location of the brightest spots are in agreement within uncertainties and can be explained by inhomogeneous exposure and uncertainties in background estimation. The same can be applied to the differences between spectral indices of Region A and Region B.

The results obtained point to the importance of investigating these kinds of sources with IACTs: while both Fermi-LAT and LHAASO brought interesting results on the lower and higher energy tails of the gamma spectra, only Cherenkov telescopes featuring higher angular resolution are able to distinguish between different contributions. We will continue investigations of this source including 
multi-component fitting and modelling with GAMERA [25] and naima [26] software.

\section{Acknowledgements}

This research made use of gammapy, ${ }^{1}$ a community-developed core Python package for TeV gamma-ray astronomy [20, 21]. The maps of molecular clouds on Fig. 4 are kindly provided by authors of Ref. [9]. The H.E.S.S. acknowledgements can be found in: https://www.mpi-hd.mpg.de/hfm/HESS/pages/ publications/auxiliary/HESS-Acknowledgements-2021.html

\section{References}

[1] H.E.S.S. collaboration, Acceleration of petaelectronvolt protons in the Galactic Centre, Nature 531 (2016) 476 [1603.07730].

[2] H.E.S.S. collaboration, Evidence of $100 \mathrm{TeV} \gamma$-ray emission from HESS J1702-420: A new PeVatron candidate, 2106.06405.

[3] HAWC collaboration, Multiple Galactic Sources with Emission Above 56 TeV Detected by HAWC, Phys. Rev. Lett. 124 (2020) 021102 [1909. 08609].

[4] Z. Cao et al., Ultrahigh-energy photons up to 1.4 petaelectronvolts from 12 -ray galactic sources, Nature 594 (2021) 33.

[5] HESS collaboration, Particle Transport within the Pulsar Wind Nebula HESS J1825-137, Astron. Astrophys. 621 (2019) A116 [1810. 12676].

[6] H.E.S.S. collaboration, An extreme particle accelerator in the Galactic plane: HESS J1826-130, Astron. Astrophys. 644 (2020) A112 [2010 . 13101].

[7] A.A. Abdo et al., TeV Gamma-Ray Sources from a Survey of the Galactic Plane with Milagro, Astrophys. J. Lett. 664 (2007) L91 [0705 . 0707].

[8] F. Aharonian, Detection of Very High Energy radiation from HESS J1908+063 confirms the Milagro unidentified source MGRO J1908+06, Astron. Astrophys. 499 (2009) 723 [0904.3409].

[9] L. Duvidovich, A. Petriella and E. Giacani, Radio study of the extended TeV source VER J1907+062, Mon. Not. Roy. Astron. Soc. 491 (2020) 5732 [1912 . 13352].

[10] S. Crestan, A. Giuliani, S. Mereghetti, L. Sidoli, F. Pintore and N. La Palombara, Multiwavelength investigation of the candidate Galactic PeVatron MGRO J1908+06, 2105.07001.

[11] J. Li, R.-Y. Liu, E. de Oña Wilhelmi, D.F. Torres, Q.-C. Liu, M. Kerr et al., Investigating the Nature of MGRO J1908+06 with Multiwavelength Observations, Astrophys. J. Lett. 913 (2021) L33 [2102 .05615].

\footnotetext{
1https://www . gammapy .org
} 
[12] HESS collaboration, The H.E.S.S. Galactic plane survey, Astron. Astrophys. 612 (2018) A1 [1804.02432].

[13] Fermi-LAT collaboration, Detection of 16 Gamma-Ray Pulsars Through Blind Frequency Searches Using the Fermi LAT, Science 325 (2009) 840 [1009. 0748].

[14] J.M. Stil, A.R. Taylor, J.M. Dickey, D.W. Kavars, P.G. Martin, T.A. Rothwell et al., The VLA Galactic Plane Survey, Astron. J. 132 (2006) 1158 [astro-ph/0605422].

[15] A.G. Lyne et al., Timing of 29 Pulsars Discovered in the PALFA Survey, Astrophys. J. 834 (2017) 137 [1608.09007].

[16] C.J. Clark et al., PSR J1906+0722: An Elusive Gamma-ray Pulsar, Astrophys. J. Lett. 809 (2015) L2 [1508 . 00779].

[17] D.A. Leahy and S. Ranasinghe, Distance and Evolutionary State of the Supernova Remnant 3C 397 (G41.1-0.3), Astrophys. J. 817 (2016) 74.

[18] R.D. Parsons and J.A. Hinton, A Monte Carlo Template based analysis for Air-Cherenkov Arrays, Astropart. Phys. 56 (2014) 26 [1403.2993].

[19] S. Ohm, C. van Eldik and K. Egberts, Gamma-Hadron Separation in Very-High-Energy gamma-ray astronomy using a multivariate analysis method, Astropart. Phys. 31 (2009) 383 [0904.1136].

[20] C. Deil, R. Zanin, J. Lefaucheur, C. Boisson, B. Khelifi, R. Terrier et al., Gammapy - A prototype for the CTA science tools, in 35th International Cosmic Ray Conference (ICRC2017), vol. 301 of International Cosmic Ray Conference, p. 766, Jan., 2017 [1709.01751].

[21] C. Nigro, C. Deil, R. Zanin, T. Hassan, J. King, J.E. Ruiz et al., Towards open and reproducible multi-instrument analysis in gamma-ray astronomy, Astron. Astrophys. 625 (2019) A10 [1903.06621].

[22] T.P. Li and Y.Q. Ma, Analysis methods for results in gamma-ray astronomy, Astrophys. J. 272 (1983) 317.

[23] L. Mohrmann, A. Specovius, D. Tiziani, S. Funk, D. Malyshev, K. Nakashima et al., Validation of open-source science tools and background model construction in $\gamma$-ray astronomy, Astron. Astrophys. 632 (2019) A72 [1910.08088].

[24] H.E.S.S. collaboration, Revealing $x$-ray and gamma ray temporal and spectral similarities in the GRB 190829A afterglow, Science 372 (2021) 1081 [2106.02510].

[25] J. Hahn, GAMERA - a new modeling package for non-thermal spectral modeling, PoS ICRC2015 (2016) 917.

[26] V. Zabalza, naima: a Python package for inference of relativistic particle energy distributions from observed nonthermal spectra, PoS ICRC2015 (2016) 922 [1509. 03319]. 


\section{Full Authors List: H.E.S.S. Collaboration}

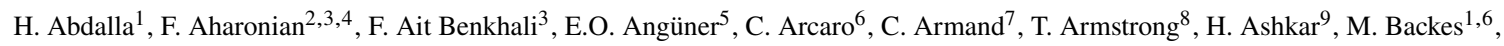
V. Baghmanyan ${ }^{10}$, V. Barbosa Martins ${ }^{11}$, A. Barnacka ${ }^{12}$, M. Barnard ${ }^{6}$, R. Batzofin ${ }^{13}$, Y. Becherini ${ }^{14}$, D. Berge ${ }^{11}$, K. Bernlöhr ${ }^{3}$, B. Bi ${ }^{15}$, M. Böttcher ${ }^{6}$, C. Boisson ${ }^{16}$, J. Bolmont ${ }^{17}$, M. de Bony de Lavergne ${ }^{7}$, M. Breuhaus ${ }^{3}$, R. Brose ${ }^{2}$, F. Brun ${ }^{9}$, T. Bulik ${ }^{18}$, T. Bylund ${ }^{14}$, F. Cangemi ${ }^{17}$, S. Caroff ${ }^{17}$, S. Casanova ${ }^{10}$, J. Catalano ${ }^{19}$, P. Chambery ${ }^{20}$, T. Chand ${ }^{6}$, A. Chen ${ }^{13}$, G. Cotter ${ }^{8}$, M. Curyło ${ }^{18}$, H. Dalgleish ${ }^{1}$, J. Damascene Mbarubucyeye ${ }^{11}$, I.D. Davidss ${ }^{1}$, J. Davies ${ }^{8}$, J. Devin ${ }^{20}$, A. Djannati-Ataï ${ }^{21}$, A. Dmytriiev ${ }^{16}$, A. Donath ${ }^{3}$, V. Doroshenko ${ }^{15}$, L. Dreyer ${ }^{6}$, L. Du Plessis ${ }^{6}$, C. Duffy ${ }^{22}$, K. Egberts ${ }^{23}$, S. Einecke ${ }^{24}$, J.-P. Ernenwein ${ }^{5}$, S. Fegan ${ }^{25}$, K. Feijen ${ }^{24}$, A. Fiasson ${ }^{7}$, G. Fichet de Clairfontaine ${ }^{16}$, G. Fontaine ${ }^{25}$, F. Lott $^{1}$, M. Füßling ${ }^{11}$, S. Funk ${ }^{19}$, S. Gabici ${ }^{21}$, Y.A. Gallant ${ }^{26}$, G. Giavitto ${ }^{11}$, L. Giunti ${ }^{21,9}$, D. Glawion ${ }^{19}$, J.F. Glicenstein ${ }^{9}$, M.-H. Grondin ${ }^{20}$, S. Hattingh ${ }^{6}$, M. Haupt ${ }^{11}$, G. Hermann ${ }^{3}$, J.A. Hinton ${ }^{3}$, W. Hofmann ${ }^{3}$, C. Hoischen ${ }^{23}$, T. L. Holch ${ }^{11}$, M. Holler ${ }^{27}$, D. Horns ${ }^{28}$, Zhiqiu Huang ${ }^{3}$, D. Huber ${ }^{27}$, M. Hörbe ${ }^{8}$, M. Jamrozy ${ }^{12}$, F. Jankowsky ${ }^{29}$, V. Joshi ${ }^{19}$, I. Jung-Richardt ${ }^{19}$, E. Kasai ${ }^{1}$, K. Katarzyński ${ }^{30}$, U. Katz $^{19}$, D. Khangulyan ${ }^{31}$, B. Khélifi ${ }^{21}$, S. Klepser ${ }^{11}$, W. Kluźniak ${ }^{32}$, Nu. Komin ${ }^{13}$, R. Konno ${ }^{11}$, K. Kosack ${ }^{9}$, D. Kostunin ${ }^{11}$, M. Kreter ${ }^{6}$, G. Kukec Mezek ${ }^{14}$, A. Kundu ${ }^{6}$, G. Lamanna ${ }^{7}$, S. Le Stum ${ }^{5}$, A. Lemière ${ }^{21}$, M. Lemoine-Goumard ${ }^{20}$, J.-P. Lenain ${ }^{17}$, F. Leuschner ${ }^{15}$, C. Levy ${ }^{17}$, T. Lohse ${ }^{33}$, A. Luashvili ${ }^{16}$, I. Lypova ${ }^{29}$, J. Mackey ${ }^{2}$, J. Majumdar ${ }^{11}$, D. Malyshev ${ }^{15}$, D. Malyshev ${ }^{19}$, V. Marandon ${ }^{3}$, P. Marchegiani ${ }^{13}$, A. Marcowith ${ }^{26}$, A. Mares ${ }^{20}$, G. Martí-Devesa ${ }^{27}$, R. $\operatorname{Mar}^{29}$, G. Maurin ${ }^{7}$, P.J. Meintjes ${ }^{34}$, M. Meyer ${ }^{19}$, A. Mitchell ${ }^{3}$, R. Moderski ${ }^{32}$, L. Mohrmann ${ }^{19}$, A. Montanari ${ }^{9}$, C. Moore ${ }^{22}$, P. Morris ${ }^{8}$, E. Moulin, J. Muller ${ }^{25}$, T. Murach ${ }^{11}$, K. Nakashima ${ }^{19}$, M. de Naurois ${ }^{25}$, A. Nayerhoda ${ }^{10}$, H. Ndiyavala ${ }^{6}$, J. Niemiec ${ }^{10}$, A. Priyana Noel ${ }^{12}$, P. O’Brien ${ }^{22}$, L. Oberholzer ${ }^{6}$, S. Ohm ${ }^{11}$, L. Olivera-Nieto ${ }^{3}$, E. de Ona Wilhelmi ${ }^{11}$, M. Ostrowski ${ }^{12}$, S. Panny ${ }^{27}$, M. Panter ${ }^{3}$, R.D. Parsons ${ }^{33}$, G. Peron ${ }^{3}$, S. Pita ${ }^{21}$, V. Poireau ${ }^{7}$, D.A. Prokhorov ${ }^{35}$, H. Prokoph ${ }^{11}$, G. Pühlhofer ${ }^{15}$, M. Punch ${ }^{21,14}$, A. Quirrenbach ${ }^{29}$, P. Reichherzer ${ }^{9}$, A. Reimer ${ }^{27}$, O. Reimer ${ }^{27}$, Q. Remy ${ }^{3}$, M. Renaud ${ }^{26}$, B. Reville ${ }^{3}$, F. Rieger ${ }^{3}$, C. Romoli ${ }^{3}$, G. Rowell ${ }^{24}$, B. Rudak ${ }^{32}$, H. Rueda Ricarte ${ }^{9}$, E. Ruiz-Velasco ${ }^{3}$, V. Sahakian ${ }^{36}$, S. Sailer ${ }^{3}$, H. Salzmann ${ }^{15}$, D.A. Sanchez ${ }^{7}$, A. Santangelo ${ }^{15}$, M. Sasaki ${ }^{19}$, J. Schäfer ${ }^{19}$, H.M. Schutte ${ }^{6}$, U. Schwanke ${ }^{33}$, F. Schüssler ${ }^{9}$, M. Senniappan ${ }^{14}$, A.S. Seyffert ${ }^{6}$, J.N.S. Shapopi ${ }^{1}$, K. Shiningayamwe ${ }^{1}$, R. Simoni ${ }^{35}$, A. Sinha ${ }^{26}$, H. Sol ${ }^{16}$, H. Spackman ${ }^{8}$, A. Specovius ${ }^{19}$, S. Spencer ${ }^{8}$, M. Spir-Jacob ${ }^{21}$, L. Stawarz ${ }^{12}$, R. Steenkamp ${ }^{1}$, C. Stegmann ${ }^{23,11}$, S. Steinmass ${ }^{3}$, C. Steppa ${ }^{23}$, L. Sun ${ }^{35}$, T. Takahashi ${ }^{31}$, T. Tanaka ${ }^{31}$, T. Tavernier ${ }^{9}$, A.M. Taylor ${ }^{11}$, R. Terrier ${ }^{21}$, J. H.E. Thiersen ${ }^{6}$, C. Thorpe-Morgan ${ }^{15}$, M. Tluczykont ${ }^{28}$, L. Tomankova ${ }^{19}$, M. Tsirou ${ }^{3}$, N. Tsuji ${ }^{31}$, R. Tuffs ${ }^{3}$, Y. Uchiyama ${ }^{31}$, D.J. van der Walt ${ }^{6}$, C. van Eldik ${ }^{19}$, C. van Rensburg ${ }^{1}$, B. van Soelen $^{34}$, G. Vasileiadis ${ }^{26}$, J. Veh ${ }^{19}$, C. Venter ${ }^{6}$, P. Vincent ${ }^{17}$, J. Vink ${ }^{35}$, H.J. Völk ${ }^{3}$, S.J. Wagner ${ }^{29}$, J. Watson ${ }^{8}$, F. Werner ${ }^{3}$, R. White ${ }^{3}$, A. Wierzcholska ${ }^{10}$, Yu Wun Wong ${ }^{19}$, H. Yassin ${ }^{6}$, A. Yusafzai ${ }^{19}$, M. Zacharias ${ }^{16}$, R. Zanin ${ }^{3}$, D. Zargaryan ${ }^{2,4}$, A.A. Zdziarski ${ }^{32}$, A. Zech ${ }^{16}$, S.J. Zhu ${ }^{11}$, A. Zmija ${ }^{19}$, S. Zouari ${ }^{21}$ and N. Żywucka ${ }^{6}$.

${ }^{1}$ University of Namibia, Department of Physics, Private Bag 13301, Windhoek 10005, Namibia

${ }^{2}$ Dublin Institute for Advanced Studies, 31 Fitzwilliam Place, Dublin 2, Ireland

${ }^{3}$ Max-Planck-Institut für Kernphysik, P.O. Box 103980, D 69029 Heidelberg, Germany

${ }^{4}$ High Energy Astrophysics Laboratory, RAU, 123 Hovsep Emin St Yerevan 0051, Armenia

${ }^{5}$ Aix Marseille Université, CNRS/IN2P3, CPPM, Marseille, France

${ }^{6}$ Centre for Space Research, North-West University, Potchefstroom 2520, South Africa

${ }^{7}$ Laboratoire d'Annecy de Physique des Particules, Univ. Grenoble Alpes, Univ. Savoie Mont Blanc, CNRS, LAPP, 74000 Annecy, France

${ }^{8}$ University of Oxford, Department of Physics, Denys Wilkinson Building, Keble Road, Oxford OX1 3RH, UK

${ }^{9}$ IRFU, CEA, Université Paris-Saclay, F-91191 Gif-sur-Yvette, France

${ }^{10}$ Instytut Fizyki Jạdrowej PAN, ul. Radzikowskiego 152, 31-342 Kraków, Poland

${ }^{11}$ DESY, D-15738 Zeuthen, Germany

${ }^{12}$ Obserwatorium Astronomiczne, Uniwersytet Jagielloński, ul. Orla 171, 30-244 Kraków, Poland

${ }^{13}$ School of Physics, University of the Witwatersrand, 1 Jan Smuts Avenue, Braamfontein, Johannesburg, 2050 South Africa

${ }^{14}$ Department of Physics and Electrical Engineering, Linnaeus University, 35195 Växjö, Sweden

${ }^{15}$ Institut für Astronomie und Astrophysik, Universität Tübingen, Sand 1, D 72076 Tübingen, Germany

${ }^{16}$ Laboratoire Univers et Théories, Observatoire de Paris, Université PSL, CNRS, Université de Paris, 92190 Meudon, France

${ }^{17}$ Sorbonne Université, Université Paris Diderot, Sorbonne Paris Cité, CNRS/IN2P3, Laboratoire de Physique Nucléaire et de Hautes Energies, LPNHE, 4 Place Jussieu, F-75252 Paris, France

${ }^{18}$ Astronomical Observatory, The University of Warsaw, Al. Ujazdowskie 4, 00-478 Warsaw, Poland

${ }^{19}$ Friedrich-Alexander-Universität Erlangen-Nürnberg, Erlangen Centre for Astroparticle Physics, Erwin-Rommel-Str. 1, D 91058 Erlangen, Germany

${ }^{20}$ Université Bordeaux, CNRS/IN2P3, Centre d'Études Nucléaires de Bordeaux Gradignan, 33175 Gradignan, France

${ }^{21}$ Université de Paris, CNRS, Astroparticule et Cosmologie, F-75013 Paris, France

${ }^{22}$ Department of Physics and Astronomy, The University of Leicester, University Road, Leicester, LE1 7RH, United Kingdom

${ }^{23}$ Institut für Physik und Astronomie, Universität Potsdam, Karl-Liebknecht-Strasse 24/25, D 14476 Potsdam, Germany

${ }^{24}$ School of Physical Sciences, University of Adelaide, Adelaide 5005, Australia

${ }^{25}$ Laboratoire Leprince-Ringuet, École Polytechnique, CNRS, Institut Polytechnique de Paris, F-91128 Palaiseau, France

${ }^{26}$ Laboratoire Univers et Particules de Montpellier, Université Montpellier, CNRS/IN2P3, CC 72, Place Eugène Bataillon, F-34095 Montpellier Cedex 5, France

${ }^{27}$ Institut für Astro- und Teilchenphysik, Leopold-Franzens-Universität Innsbruck, A-6020 Innsbruck, Austria

${ }^{28}$ Universität Hamburg, Institut für Experimentalphysik, Luruper Chaussee 149, D 22761 Hamburg, Germany 
${ }^{29}$ Landessternwarte, Universität Heidelberg, Königstuhl, D 69117 Heidelberg, Germany

${ }^{30}$ Institute of Astronomy, Faculty of Physics, Astronomy and Informatics, Nicolaus Copernicus University, Grudziadzka 5, 87-100 Torun, Poland

${ }^{31}$ Department of Physics, Rikkyo University, 3-34-1 Nishi-Ikebukuro, Toshima-ku, Tokyo 171-8501, Japan

${ }^{32}$ Nicolaus Copernicus Astronomical Center, Polish Academy of Sciences, ul. Bartycka 18, 00-716 Warsaw, Poland

${ }^{33}$ Institut für Physik, Humboldt-Universität zu Berlin, Newtonstr. 15, D 12489 Berlin, Germany

${ }^{34}$ Department of Physics, University of the Free State, PO Box 339, Bloemfontein 9300, South Africa

${ }^{35}$ GRAPPA, Anton Pannekoek Institute for Astronomy, University of Amsterdam, Science Park 904, 1098 XH Amsterdam, The Netherlands

${ }^{36}$ Yerevan Physics Institute, 2 Alikhanian Brothers St., 375036 Yerevan, Armenia 